\title{
A Novel Approach to Study the Effects of Anesthesia on Respiratory Signals by using the EEG Signals
}

\author{
Mohd Suhaib Kidwai*, S. Hasan Saeed \\ Department of Electronics and Communication Engineering, Integral University, India
}

\begin{tabular}{l} 
Article Info \\
\hline Article history: \\
Received Feb 20, 2017 \\
Revised Jun 27, 2017 \\
Accepted Jul 20, 2017 \\
\hline
\end{tabular}

\section{Keywords:}

Anesthesia

EEG Machine

Order patterns

Recurrence

Synchronization Index

\begin{abstract}
General anesthesia plays a crucial role in many surgical procedures. It is a drug-induced, reversible state characterized by unconsciousness, antinociception or analgesia, immobility and amnesia. On rare occasions, however, the patient can remain unconscious longer than intended, or may regain awareness during surgery. There are no precise measures for maintaining the correct dose of anesthetic, and there is currently no fully reliable instrument to monitor depth of anesthesia. Although a number of devices for monitoring brain function or sympathetic output are commercially available, the anesthetist also relies on clinical assessment and experience to judge anesthetic depth. The undesirable consequences of overdose or unintended awareness might in principle be ameliorated by improved control if we could understand better the changes in function that occur during general anesthesia. Coupling functions prescribe the physical rule specifying how the inter-oscillator interactions occur. They determine the possibility of qualitative transitions between the oscillations, e.g. routes into and out of phase synchronization. Their decomposition can describe the functional contribution from each separate subsystem within a single coupling relationship. In this way, coupling functions offer a unique means of describing mechanisms in a unified and mathematically precise way. It is a fast growing field of research, with much recent progress on the theory and especially towards being able to extract and reconstruct the coupling functions between interacting oscillations from data, leading to useful applications in cardio respiratory interactions.

In this paper, a novel approach has been proposed for detecting the changes in synchronism of brain signals, taken from EEG machine. During the effect of anesthesia, there are certain changes in the EEG signals. Those signals show changes in their synchronism. This phenomenon of synchronism can be utilized to study the effect of anesthesia on respiratory parameters like respiration rate etc, and hence the quantity of anesthesia can be regulated, and if any problem occurs in breathing during the effect of anesthesia on patient, that can also be monitored
\end{abstract}

Copyright $@ 2017$ Institute of Advanced Engineering and Science. All rights reserved.

\section{Corresponding Author:}

Mohd Suhaib Kidwai,

Department of Electronics and Communication Engineering,

Integral University,

Lucknow,U.P, India

Email: dan_kdw@rediffmail.com

\section{INTRODUCTION}

Every function of the human body is being controlled by brain, and as such there is a certain kind of synchronism between brain and various voluntary and involuntary processes of body. When this synchronism is lost it becomes a disorder, for example epileptic fits, breathlessness etc [1]. 
In this paper focus is there, on understanding the synchronism between the brain and respiratory biomedical signals and hence trying to understand what effect does anesthesia delivery has, on the patient's respiratory rate.The signals are taken from EEG machine and are studied for the corresponding respiratory rate changes during dissemination of anesthesia to the patient during surgeries. This work mainly proposes an algorithm, which utilizes the concept of recurrence to analyze the synchronism between the brain and breathing phenomenon.

The algorithm uses the concept of recurrence and tells the degree of coupling or synchronism between the brain signals (EEG signals) and respiration rate. Hence, it will help the anesthetist to monitor the effect of anesthesia on the patient's vital parameters and it can also be used to study about the quantity of anesthesia that can be given to the patient. follows:

But before proceeding to the main work, let's focus on the various other methods. They are as

\subsection{Decomposition Model (Based on Coupling)}

The form of a coupling function depends on the differing contributions from individual oscillations. Changes in form may depend predominantly on only one of the phases (along one-axis), or they may depend on both phases, often resulting in a complicated and intuitively unclear dependence. This demonstrates the need for a model able to distinguish the individual functional contributions to a coupling.

This model mainly takes phase into consideration, though amplitude dynamics can also be taken into consideration. The coupling between the various signals or the recurrences is found out and disorders are diagnosed on the basis of coupling strength [3-6].

Previous coupling treatments, including the cross-frequency coupling in neuroscience, have focused on the net coupling in one direction. In this model, we decompose the net coupling into two components depending on their functional roles: the direct and the indirect couplings. Direct-coupling describes the influence of the direct (unidirectional) driving that one oscillator exerts on the other. Arguably, it is the most studied interaction in physiology, often linked to modulation mechanisms. The second component, indirectcoupling, often called common-coupling, and depends on the shared contributions of the two oscillations. The indirect coupling also includes the diffusive coupling given with the phase difference terms. The mechanism behind this coupling component or process, although we present the model in relation to phase dynamics, a similar functional decomposition of the couplings can also be applied to amplitude dynamics.

In terms of the general theory of phase dynamics and equation, the coupling function q1 $(\phi 1, \phi 2)$ can be expressed as the product of two functions

$$
\mathrm{q} 1(\phi 1, \phi 2)=\mathrm{P} 1(\phi 1) \mathrm{P} 2(\phi 2)
$$

where $\mathrm{P} 1(\phi 1)$ is the phase response curve (PRC) of the first oscillator and shows how it responds to external perturbations, while $\mathrm{P} 2(\phi 2)$ is the perturbation function through which the second oscillator acts on the first one [7-8].

\subsection{Signal processing and statistical analysis}

The signals were first inspected visually, followed by automated artifact removal by interpolation. Data from subjects whose signals had many artifacts were disregarded and not analyzed. The cross-frequency intervals were estimated by standard digital filtering procedures, including a FIR filter followed by a zerophase digital filtering procedure to ensure that no time or phase lags were introduced by the filtering. The boundaries of the intervals extracted from the EEG signal were $\delta=0.8-4 \mathrm{~Hz}, \theta=4-7.5 \mathrm{~Hz}, \alpha=7.5-14 \mathrm{~Hz}$, $\beta=14-22 \mathrm{~Hz}$ and $\gamma=22-100 \mathrm{~Hz}$; the interval extracted from the respiration signal was $\mathrm{r}=0.145-0.6 \mathrm{~Hz}$; and the extraction of the heart activity from the ECG signal was $\mathrm{h}=0.6-2 \mathrm{~Hz}$. Wavelet power and coherence analyses, together with further clinical interpretation, will be presented elsewhere. For the EEG oscillations special care was taken in dealing with frequency spillage between intervals, heart artifacts and power line artifacts [7-8].

Then the coupling between these obtained ECG signals and those obtained from EEG machine is studied and compared with the standard values of various statistical parameters, which are obtained from the healthy person.

Following parameters can be used to study the recurrence pattern in the biomedical signals:

1. Determinism(DET): These measures are based on the histogram $P(l)$ of diagonal lines of length $l$. Processes with uncorrelated or weakly correlated, stochastic or chaotic behavior cause none or very short diagonals, whereas deterministic processes cause longer diagonals and less single, isolated recurrence points. Therefore, the ratio of recurrence points that form diagonal structures 
(of minimum length $l \mathrm{~min}$ ) to all recurrence points is introduced as a measure for determinism (or predictability) of the system:

$\mathrm{DET}=\sum_{\text {lmin. }}^{N} l P(l) / \sum_{l=1}^{N} l P(l)$

2. A diagonal line of length $l$ means that a segment of the trajectory is rather close during 1 time step to another segment of the trajectory at a different time; thus, these lines are related to the divergence of the trajectory segments. The average diagonal line length is the average time that two segments of the trajectory are close to each other, and can be interpreted as the mean prediction time [9-10].

$$
\mathrm{L}=\sum_{l m i n}^{N} l P(l) / \sum_{l m i n .}^{N} P(l)
$$

3. Measures based on vertical lines: These measures are based on the histogram $\mathrm{P}(\mathrm{v})$ of vertical lines of length $\mathrm{v}$. The ratio between the recurrence points forming the vertical structures and the entire set of recurrence points is called laminarity [10-11].

$$
\mathrm{LAM}=\sum_{v \min }^{N} v P(v) / \sum_{v=1}^{N} v P(v)
$$

The computation of LAM is realized for those $v$ that exceed a minimal length $v$ min in order to decrease the influence of the tangential motion. LAM represents the occurrence of laminar states in the system without describing the length of these laminar phases. It will decrease if the RP consists of more single recurrence points than vertical structures.

The average length of vertical structures is called trapping time (TT)

$$
\mathrm{LAM}=\sum_{v \min .}^{N} v P(v) / \sum_{v \min .}^{N} P(v)
$$

\section{RESEARCH METHOD}

The mathematical concept of recurrence is the crux of the algorithm. The EEG signals from various channels of EEG machine are taken as an input which will be used by the program in MATLAB. This program will be based on the proposed algorithm and basically compares the synchronism between the EEG signals of any two channels. This comparison is done by using the concept of recurrence.

Second thing is to find the degree of recurrence which is found out by using the formula of synchronization index $\rho \pi$ [13-14].

The advantage of using synchronization index in this algorithm is that it serves two purposes, firstly it helps in understanding the synchronization between the two signals from EEG channels and secondly it allows the user to find the degree of synchronization.

Using the plot command of MATLAB, this synchronization index can be shown in form of graphs .If the graph shows increasing pattern, it shows high amount of synchronization between the two signals else the low synchronization.

Figure 1 is the diagrammatic representation of the proposed algorithm for better visualization and understanding: 


\section{Block Diagram}

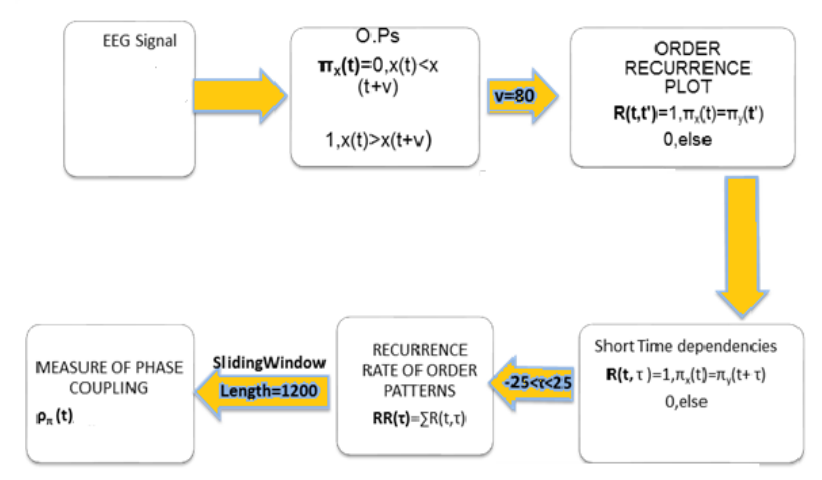

Figure 1. The block diagram of the proposed algorithm

The steps of algorithm, whose block diagram is depicted as Figure 1, can be defined as follows:

a. The signals from the channels of EEG machines are taken and put in a form of array so that it can be used as an input.

b. The signals from the channels of EEG machines are taken and put in a form of array so that it can be used as an input in MATLAB program based on this algorithm.

c. pie $\mathrm{x}\left(\pi_{x}\right)$ refers to the order pattern and it is found out by comparing samples by their respective values at different intervals. Similarly pie $\mathrm{y}\left(\pi_{\mathrm{y}}\right)$ is found.

d. Once we have the values of pie $\mathrm{x}$ and pie $\mathrm{y}$ in form of matrices next step is to find order pattern. It is found as follows:

$$
\begin{aligned}
\operatorname{ORP}(t, \tau)= & 1 \text { if } \pi_{\mathrm{x}}(\mathrm{t})=\pi_{\mathrm{y}}(\mathrm{t}+\tau) \\
& 0, \text { otherwise }
\end{aligned}
$$
formulas

Next step is to find out the recurrence rate and its normalized value. This is done using the following

$$
\begin{aligned}
& \operatorname{RR}(\tau)=\sum_{t} R(t, \tau) \\
& \operatorname{rr}(\tau)=\operatorname{RR}(\tau) / \sum \operatorname{RR}(\tau)
\end{aligned}
$$

Then finally a parameter called "synchronization index" $\left(\rho_{\pi}\right)$ is found and plotted. The formula for synchronization index is as follows:

$$
\rho_{\pi}=1-\left(\frac{-\sum_{\tau \min }^{\tau \max } \operatorname{rr}(\tau) \ln \operatorname{rr}(\tau)}{\ln (\tau \max -\tau \min )}\right)
$$

This parameter " $\rho_{\pi}$ ", when plotted between its two channels using MATLAB, will show either high, moderate, low or no synchronization between the signals taken from different channels of MATLAB.

Based on the value of synchronization index, one can compare the synchronism between brain signals that controls the respiratory functions of body, to the value of synchronization index obtained from the healthy person(reference values).

This way it will become easier to understand how anesthesia delivered to a person, is affecting its respiratory function and the amount of dose needed.

The only precaution which may be required in this entire process is correct placement of EEG electrode, only at that position of brain, which controls the respiratory signals.

The appealing factor of this algorithm is that it is very simple and the method developed using this algorithm will be a universal method which will be helpful in studying, detecting or diagnosing all those neurological disorders, that manifest themselves into changes in the pattern of EEG signals.

Moreover, this algorithm may be used for detecting diseases which manifest themselves in changes in ECG and EMG signals also, with slight changes. So, in a way this proposed algorithm can be used as a 
basic framework which will pave the way for studying, and diagnosing diseases that manifest themselves into some form of biomedical signals, like ECG and EMG signals.

Now the proposed algorithm is compred with the other methods mentioned above, as follows:

a. Comparison with the Decomposition Model: Decomposition model mainly focuses on finding the synchronism between the two or more signals using the phase, as a parameter.Now the problem with this method is that phase decomposition techniques are complex and often require associated circuitry which increases the overall cost of the device or the machine which will use the algorithm based on this model.Contrary to this,the algorithhem proposed in this paper, will find the synchronism between the two signals by using the simple mathematical concept of recurrences. The proposed algorithm simply uses the generalized formula of "Synchronization Index $\left(\rho_{\pi}\right)$ ". This approach ensures that no additional circuitry is involved as there is no need to extract any phase related information from the signal.So this method is cost effective and simple.

b. Comparison with model based on signal processing and statistical analysis: The various approaches which use signal processing methods generally use the algorithms like KNN algorithm etc [15-16].

These algorithms are very application specific, so suppose if they are designed for a specific application, like for detecting epileptic seizure, then if that same method is used for finding say, Alzheimer, then lot of changes needs to be done in source code and source program. But if we use the synchronization index as a parameter to find the neurological disorder, it will serve as a uniform and generalized method, which can be used to detect any neurological disorder that manifest itself in the synchronization of signals. Similarly, if we compare the proposed algorithm with methods based on statistical analysis, we will see that methods based on statistical analysis use various parameters like determinism, laminarity etc to determine synchronism, while the proposed algorithm only uses a single parameter "synchronization Index", so obviously the latter approach is simpler as compared to the former one.

\section{RESULTS AND ANALYSIS}

Simplicity and robustness is the crux of the proposed algorithm.From the comparison done in the previous section we can easily make out that this algorithm has a generalized approach in determining any disorder which manifests itself in any kind of synchronism in biomedical signals.

Since the algorithm has a generalized approach towards any kind neurological disorder, the program which will be developed based on this approach can be used for detecting any kind of neurological disease that manifests itself in synchronization between signals.

\section{CONCLUSION}

This algorithm is simple and with slight changes, it can be applied to diagnose different neurological disorders like epilepsy, alzheimer's disease, parkinson's syndrome etc, since most of the neurological disorders exhibit disturbance in regular, synchronized patterns of biomedical signals like EEG and ECG signals. That disturbance or change in the signal pattern can be visualized using the above proposed algorithm.

Moreover this algorithm also paves the way for the development of software based techniques which can lead to the simple yet effective diagnosis of neurological disorders and also explains the complex recurrence patterns between various activities of the human body.

\section{REFERENCES}

[1] A. Bahraminasab, F. Ghasemi, A. Stefanovska, P.V.E. McClintock, H. Kantz, "Direction of Coupling from Phases of Interacting Oscillators: A Permutation Information Approach”, PHYSICAL REVIEW LETTERS, 100, 084101 (2008).

[2] Andreas Groth, "Visualization of Coupling in Time Series by Order Recurrence Plots", PHYSICAL REVIEW E 72, 046220 (2005)

[3] A. Pikovsky, M. Rosenblum, and J. Kurths, "Synchronization- A Universal Concept in Nonlinear Sciences", Cambridge University Press, Cambridge, England, (2001).

[4] A. Stefanovska, H. Haken, P. V. E. McClintock, M. Hozic, F. Bajrovic, and S. Ribaric, "Reversible Transitions between Synchronization States of the Cardiorespiratory System".

[5] Cardio respiratory System Phys. Rev. Lett.85, 4831(2000).Sacred Heart HealthCare System, Ambulatory Electroencephalography (EEG) [Online]. Available:http://www.shh.org/hospital-services/ neurodiagnostictesting/ambulatory.asp, Mar. 22, 2014 
[6] M. Niknazar, S. R. Mousavi, B. Vosoughi Vahdat, and M. Sayyah, "A New Framework Based on Recurrence Quantification Analysis for Epileptic Seizure Detection", IEEE Journal of Biomedical and Health Informatics; vol. 17(3), May 2013.

[7] J. Engel, T. Pedley, J. Aicardi, M. Dichter, and S. Mosh. "Epilepsy: a Comprehensive Textbook". Lippincott Williams \& Wilkins, Jan 2007.

[8] B. Litt, A. D’Alessandro, R. Esteller, J. Echauz, and G. Vachtsevanos, "Translating Seizure Detection, Prediction and Brain Stimulation Into Implantable Devices for Epilepsy", In Conference Proceedings in the 1st International IEEE EMBS Conference on Neural Engineering, pages 485 - 488, Feb 2003.

[9] B. Litt, and K. Lehnertz, "Seizure Prediction and the Preseizure Period", Current Opinion in Neurology, 15(2):173177, Apr 2002.

[10] S. Sanei, and J. Chambers. "EEG Signal Processing”, Wiley-Interscience, 2007.

[11] B. Schelter, M. Winterhalder, T. Maiwald, A. Brandt, A. Schad, J. Timmer, and A. Schulze-Bonhage. "Do FalsePredictions of Seizures Depend on the State of Vigilance?”, Epilepsia, 47(12):20582070, Nov 2006.

[12] R. Schuyler, A. White, K. Staley, and K. Cios, "EpilepticSeizure Detection", Engineering in Medicine and Biology Magazine, IEEE, 26(2):74 - 81, Mar 2007.

[13] B. Shneker, and N. Fountain. Epilepsy. Disease-a-Month, 49(7):426-478, Jul 2003.

[14] M Winterhalder, T Maiwald, and H Voss, "The Seizure Prediction Characteristic: a General Framework to Assess Mand Compare Seizure Prediction Methods", Epilepsy and Behavior, Jan 2003.

[15] Mohammad Hossein Doost Mohammadi, "Improved Denoising Method for Ultrasonic Echo with Mother Wavelet Optimization and Best Basis Selection”, IJECE, vol 6 issue 6, Deceember 2016.

[16] Neethu Mohan, "Modified Variational Mode Decomposition for Power Line Interference Removal in ECG Signals", IJECE, vol 6 no 1, February 2016.

\section{BIOGRAPHIES OF AUTHORS}
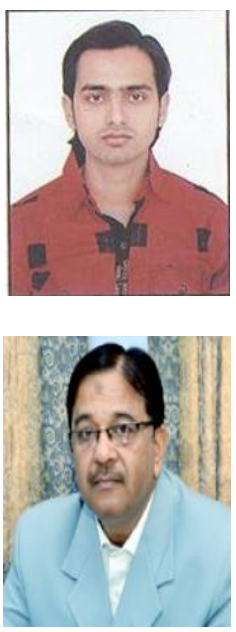

Mohd Suhaib Kidwai, is a research scholar,in the Department of Electronics and Communication,Integral University.He has interest in Digital Electronics,Signal Processing and Biomedical Electronics
Prof. S.Hasan Saeed is currently working as Professor \& Head of the Department in Electronics \& Communication Engineering department, Faculty of Engineering at Integral University. He did his B.Tech in Electrical Engineering from Zakir HussainCollege of Engineering and Technology, campus college of Aligarh Muslim University (AMU), Aligarh, Uttar Pradesh in year 1992 and received $\mathrm{PhD}$ from Integral University, Lucknow in year 2010. 